\title{
Acute Encephalopathy in a Child with Coronavirus Disease-2019 Infection
}

\author{
Aarti Kinikar ${ }^{1}$, Rajesh Kulkarni ${ }^{2}$, Uday Rajput ${ }^{3}$, Rajesh Karyakarte ${ }^{4}$
}

\begin{abstract}
Coronavirus disease-2019 (COVID-19) is a global health crisis. Children account for 1-5\% of diagnosed COVID-19 cases with relatively mild presentation and less mortality compared to adults. While patients typically present with fever, shortness of breath, and cough, neurologic manifestations have been reported, mainly in adults. We report a case of COVID-19-associated encephalopathy in an 11-year-old child who presented with altered sensorium and seizures.
\end{abstract}

Keywords: Atypical, Children, COVID-19, Encephalopathy, SARS-CoV-2.

Pediatric Infectious Disease (2020): 10.5005/jp-journals-10081-1244

Coronavirus disease-2019 (COVID-19) is a global health crisis. Children account for $1-5 \%$ of diagnosed COVID-19 cases with relatively mild presentation and less mortality compared to adults. ${ }^{1}$ While patients typically present with fever, shortness of breath, and cough, neurologic manifestations have been reported, mainly in adults. ${ }^{2}$ We report a case of COVID-19-associated encephalopathy in an 11-year old child who presented with altered sensorium and seizures.

Our patient was an 11-year old boy who premorbidly had intellectual disability and a single episode of generalized seizures at the age of 4 years, but was never on antiepileptics. Details of his past history or investigations for intellectual disability were not available as the child stayed with his aunt after death of both parents (when child was 3 years old). He presented with a 2-day history of fever and loose motions 2 days prior to admission. The child developed altered mental status and had multiple episodes of generalized tonic clonic (GTC) seizures 12 hours before admission to our hospital (after being denied admission by two hospitals).

On admission to our hospital, he had severe tachypnea and active GTC seizures. The hospital protocol for pediatric status epilepticus was followed and his seizures were controlled after about 15 minutes (lorazepam, fosphenytoin, and levetiracetam were used). The child's Glasgow coma scale on admission was $6 / 15$ and he was immediately intubated and ventilated. He had bradycardia (heart rate 60/minute), hypertension $(130 / 90 \mathrm{~mm} \mathrm{Hg}$ ), tachypnea (respiratory rate 52/minute), and bilateral mid-dilated pupils unresponsive to light. Chest auscultation did not reveal any foreign sounds. The pulse oximeter showed oxygen saturation $\left(\mathrm{SpO}_{2}\right)$ of $92 \%$. The ECG showed sinus bradycardia.

Initial laboratory workup revealed severe metabolic acidosis ( $\mathrm{pH} 6.8, \mathrm{PaO}_{2} 68 \mathrm{~mm} \mathrm{Hg}, \mathrm{PaCO}_{2} 15 \mathrm{~mm} \mathrm{Hg}$ with bicarbonate of 3 $\mathrm{mEq} / \mathrm{L}$ ) with deranged renal functions (BUN: $136 \mathrm{mg} / \mathrm{dL}$, creatinine $14 \mathrm{mg} / \mathrm{dL}$ ) and liver function (ALT: $240 \mathrm{U} / \mathrm{L}, \mathrm{AST}: 340 \mathrm{U} / \mathrm{L}, \mathrm{PT}$ INR 2.1, bilirubin $1.4 \mathrm{mg} / \mathrm{dL}$ ).There was hyperglycemia (RBS $453 \mathrm{mg} / \mathrm{dL}$ ) with grossly elevated serum amylase ( $979 \mathrm{U} / \mathrm{L})$; HbA1c was $4.8 \%$ indicating acute hyperglycemia probably due to pancreatitis. There was hyponatremia (serum sodium $127 \mathrm{mEq} / \mathrm{L}$ ). Hemogram revealed hemoglobin of $9.1 \mathrm{~g} / \mathrm{dL}$, platelet count of 90,000 with white cell count of $17.6 \times 10^{9}$, and the neutrophil lymphocyte ratio of 8.8 (neutrophil $71 \%$, lymphocytes $9 \%$ ). $C$ reactive protein (CRP) was

\footnotetext{
${ }^{1-3}$ Department of Pediatrics, BJ Government Medical College, Pune, Maharashtra, India

${ }^{4}$ Department of Microbiology, BJ Government Medical College, Pune,
} Maharashtra, India

Corresponding Author: Rajesh Kulkarni, Department of Pediatrics, BJ Government Medical College, Pune, Maharashtra, India, Phone: +91 9224799523, e-mail: docrajesh75@yahoo.com

How to cite this article: Kinikar A, Kulkarni R, Rajput U, et al. Acute Encephalopathy in a Child with Coronavirus Disease-2019 Infection. Pediatr Inf Dis 2020;2(2):62-63.

Source of support: Nil

Conflict of interest: None

positive $(3.6 \mathrm{mg} / \mathrm{dL})$; however, procalcitonin was not elevated $(0.4$ $\mathrm{ng} / \mathrm{mL})$. Serum ferritin was high $(2400 \mathrm{ng} / \mathrm{mL})$ and D-dimers were elevated $(3.5 \mathrm{mg} / \mathrm{dL})$. Chest X-ray showed mild right parahilar and lower-zone infiltration. Creatine kinase-myocardial band (CK-MB) was elevated ( $99 \mathrm{IU} / \mathrm{L})$ suggestive of myocardial injury.

SARS-CoV-2 real-time reverse transcriptase ( $r R T)-P C R$ was positive. Respiratory pathogen polymerase chain reaction (PCR) panel was negative. The cerebrospinal fluid (CSF) analysis did not show any cells. Tests for SARS-CoV-2 and standard meningitis/ encephalitis PCR panel on CSF were negative. Blood and urine cultures were sterile. We could not obtain neuroimaging due to the critical condition of the patient and bedside EEG could not be done. NS-1 and rapid malaria test were negative. The HIV test was nonreactive. Ultrasound kidney ureter bladder (KUB) showed bilateral raised renal echogenicity suggestive of acute medical renal disease.

Patient was given IV boluses of normal saline, sodium bicarbonate infusion, insulin drip (in view of hyperglycemia), antibiotic (meropenem), inotropes, steroids, enaxaparin, oseltamivir, and acyclovir. Hemodialysis was done, which reduced the creatinine to $11.5 \mathrm{mg} / \mathrm{dL}$. However, despite all measures, child succumbed within 24 hours of admission to COVID-19 PICU.

We considered a differential diagnosis of acute necrotizing encephalopathy (ANE) due to SARS-CoV-2 infection, uremic encephalopathy, and septic encephalopathy. Additionally, possibility of status epilepticus due to an abnormal brain was 
considered (in view of premorbid history intellectual disability and single episode of seizure in past). Acute necrotizing encephalopathy is a rare complication of influenza and other viral infections and has been related to intracranial cytokine storms, which result in blood-brain barrier breakdown, but without direct viral invasion or parainfectious demyelination. ${ }^{3}$ Accumulating evidence suggests that a subgroup of patients with severe COVID-19 might have a cytokine storm syndrome which our patient had. ${ }^{4}$ Acute necrotizing encephalopathy has been predominantly described in the pediatric population. The most characteristic imaging feature includes symmetric, multifocal lesions with invariable thalamic involvement. ${ }^{5}$ Unfortunately, neuroimaging could not be obtained in our patient and CSF was negative for SARS-CoV2; hence, we could not prove the diagnosis of COVID encephalopathy. However, there was a strong temporal association of encephalopathy and COVID-19 infection in our patient.

The mechanism by which COVID-19 affects the brain is not known. Direct brain infection and/or an autoimmune process may also be possible. Because the virus binds the surface spike protein to the human angiotensin-converting enzyme 2 receptor (ACE-2), and the fact that ACE- 2 is present in the brain vascular endothelium, a vascular process with clotting and infarction may also be possible and this not mechanism may be possible in our patient. ${ }^{6-8}$

Our patient had multiorgan dysfunction (encephalopathy/ pancreatitis/acute kidney injury/coagulopathy/liver dysfunction/ myocarditis) with evidence of cytokine storm (high ferritin, D dimer, CRP, CPK MB) and fulfilled WHO criteria of hyperinflammatory syndrome. There is one pediatric case report from India of hyperinflammatory syndrome treated successfully with tocilizumab and IVIG. ${ }^{9}$ In our case, delayed presentation of the child to our hospital probably contributed to the fatal outcome.

Atypical presentations of COVID-19 in children include COVID toes and hyperinflammatory syndrome. Neurological presentation with seizures is rare. ${ }^{10}$ This is the first reported case of COVID-19associated encephalopathy from India in pediatric population. As the number of patients with COVID-19 increases worldwide, pediatricians should be aware of atypical presentation with altered mental status and seizures and presentation as hyperinflammatory shock with multiorgan dysfunction among children. It may be necessary to screen children presenting with encephalopathy or encephalitis for COVID-19 as infected patients require special precautions to prevent further spread.

\section{References}

1. Wang D, Hu B, Hu C, et al. Clinical characteristics of 138 Hospitalized patients with 2019 novel coronavirus infected pneumonia in Wuhan, china. JAMA 2020;323(11):1061-1069. DOI: 10.1001/jama.2020. 1585.

2. Mao L, Wang M, Chen S, et al., Neurological Manifestations of Hospitalized Patients with COVID-19 in Wuhan, China: a retrospective case series study 2020.

3. Rossi A. Imaging of acute disseminated encephalomyelitis. Neuroimaging Clin N Am 18(1):149-161. DOI: 10.1016/j.nic.2007. 12.007.

4. Mehta P, McAuley DF, Brown M, et al. COVID-19: consider cytokine storm syndromes and immunosuppression. Lancet 2020;395(10229):1033-1034. DOI: 10.1016/S0140-6736(20)30628-0.

5. Wong AM, Simon EM, Zimmerman RA, et al. Acute necrotizing encephalopathy of childhood: correlation of MR findings and clinical outcome. AJNR Am J Neuroradiol 2006;27(9):1919-1923.

6. Clerkin KJ, Fried JA, Raikhelkar J, et al. Coronavirus disease 19 (COVID19) and cardiovascular disease. Circulation 2020;141:1648-1655. DOI: 10.1161/CirculationAHA 120.046941.

7. Zhang Y, Xiao M, Zhang S, et al. Coagulopathy and antiphospholipid antibodies in patients with COVID-19. N Engl J Med 2020;382(17):e38. DOI: 10.1056/NEJMc2007575.

8. Jiang T, Gao L, Lu J, et al. ACE2-Ang-(1-7)-Mas axis in brain: a potential target for prevention and treatment of ischemic stroke. Curr Neuropharm 2013;11(2):209-217. DOI: 10.2174/1570159X11311020007.

9. Balasubramaniam S, Nagendran T, Ramchandran B, et al. Hyperinflammatory syndrome in a child with COVID-19 treated successfully with intravenous immunoglobulin and tocilizumab. Indian Pediatr 2020. Available from: https://www.indianpediatrics.net/ COVID29.03.2020/CCL-00180.pdf Accessed June 09, 2020.

10. Dugue R, Cay-Martínez KC, Thakur KT, et al. Neurologic manifestations in an infant with COVID-19. Neurology 2020;94(24):1100-1102. DOI: 10.1212/WNL.0000000000009653. 